\title{
Reducing Number of Classifiers in DAGSVM Based on Class Similarity
}

\author{
Marcin Luckner \\ Warsaw University of Technology, Faculty of Mathematics and Information Science, \\ pl. Politechniki 1, 00-661 Warsaw, Poland \\ mluckner@mini.pw .edu .pl \\ http://www.mini.pw.edu.pl/ lucknerm/en/
}

\begin{abstract}
Support Vector Machines are excellent binary classifiers. In case of multi-class classification problems individual classifiers can be collected into a directed acyclic graph structure DAGSVM. Such structure implements One-Against-One strategy. In this strategy a split is created for each pair of classes, but, because of hierarchical structure, only a part of them is used in the single classification process.

The number of classifiers may be reduced if their classification tasks will be changed from separation of individual classes into separation of groups of classes. The proposed method is based on the similarity of classes. For near classes the structure of DAG stays immutable. For the distant classes more than one is separated with a single classifier. This solution reduces the classification cost. At the same time the recognition accuracy is not reduced in a significant way. Moreover, a number of SV, which influences on the learning time will not grow rapidly.
\end{abstract}

Keywords: Classification, Directed Acyclic Graph, Support Vector Machines, One-Against-One.

\section{Introduction}

Support Vector Machines [11] are excellent binary classifiers, which can also be applied to multi-class problems. One-step solutions [4] are mainly theoretical [6]. Instead, an ensemble of SVMs is used. There are two main strategies for that $6[1]$.

The first, One-Against-All [3] creates a classifier for each recognized class. The classifier splits a data space between the class and the rest of classes. In the $n-$ classes case the method needs $n$ classifiers. The method can also be implemented in the form of a decision tree [2. Then, on each level of the tree one class is separated with the rest. This implementation requires only $n-1$ classifiers and the average decision process uses $n-1 / 2$ classifiers.

The second method is One-Against-One [7. The method creates a classifier for each pair of classes. In comparison to the One-Against-All strategy the recognition rate grows, the same as the number of created classifiers. In the case of $n$ classes the method needs $n(n-1) / 2$ classifiers. The method can also 
be implemented in the form of a directed acyclic graph [8]. Then only $n-1$ classifiers have to be used in the classification process. However, this number is constant and independent of a recognizing class. The problem of decrease of the average classification cost is discussed in the section 2

The aim of this paper is to propose a method for a reduction of classifiers used by the DAGSVM without a significant decrease of the recognition rate. For that the classifiers for a pair of classes will be only created for the similar classes. If the class is separated from the rest of classes then the One-Against-All strategy will be used instead. The creation of the graph with reduced number of classifiers is presented in the section 3 .

The graph created in the proposed way will be tested on the handwritten digits recognition task. The results will be compared with the One-Against-All and One-Against-One strategies. The test and its results are presented in the section 4 .

\section{DAGSVM and Reduction of Classifiers}

The DAGSVM has proved it's advantages in many tests 612]. The DAGSVM implements the One-Against-One strategy. The SVM classifier is created for each pair of recognized classes. In each step of the classification algorithm one class is rejected. The second one is compared with a next class. The algorithm terminates when the single remaining class is not rejected. In the case of $n^{-}$ classes recognition task, such classification path needs $n-1$ classifiers. For the $n$ possible results the global number of classifiers, without repetition, is $n(n-1) / 2$.

Because a part of classifiers will be the same for different classification paths and the order of comparison in the fixed path is not important, the classifiers can be collected in a directed acyclic graph. Then, a single classification process needs only $n-1$ classifiers, but this number is constant.

The average number of used classifiers can be reduced when the degenerated tree structure is used. Then the One-Against-All strategy is applied. Despite of reduction of trained classifiers the learning time may grow, because each single classifier solves now a more complex problem [1].

The solution, which lies somewhere between is an Adaptive Directed Acyclic Graph [5]. ADAG uses a tournament tree to reduce the number of classifiers by half on each level of the tree. For that purpose some of the classifiers splits the space between groups of classes instead of single classes.

A different approach is presented in the paper [13, where the gravity of the classifiers is estimated on the base of the probability of positive samples, while the trivial classifiers are ignored.

In this paper the reduced number of classifiers will be achieved by replacing of the splits between classes by the splits between groups. However, the replaced classifiers will be selected on the base of the similarity between classes. Details are given in the next section. 


\section{Reduction Based on Class Similarity}

For the reduction of the number of used classifiers a new structure will be proposed. The structure will also be a directed acyclic graph, therefore some definition about graphs [12] will be formed in the section 3.1. The graph will be based on a similarity. Various definition of the similarity based on a distance are presented in the section 3.2. A structure of the graph is created by grouping of the nearest classes. An algorithm, which builds the graph is presented in the section 3.3. Finally, all nodes of the graph, which are not leaves, have to be connected with the classifiers. In the section 3.4 classification tasks for the individual classifiers are defined.

\subsection{Preliminaries}

In the presented concept a directed acyclic graph $G$, which is given by the set of nodes $V(G)$ and the set of edges $E(G)$, is equated with the set of classes. The graph with a single node is an equivalent of a single class $C_{i}$, where $i=1 \ldots n$ is an index of recognized classes. When two classes $C_{i}$ and $C_{j}$, from different nodes, are joined as leaves of the same tree then the tree is equated with the set $\left\{C_{i}, C_{j}\right\}$. The tree, which groups all equivalents of recognized classes as the leaves is equated with the set $\bigcup_{i=1}^{n} C_{i}$.

A directed acyclic graphs can be presented as a tree. In such case the number of leaves exceeds $n$, but some of them will represent the same class. The higher number of leaves increases also the number of nodes on higher levels. Still, those nodes will also be duplicated. For a simplification, in the subsequent deliberation, such tree will be describes as a graph with the leaves $L(G)$ defined as a set of nodes without successors

$$
L(G)=\left\{v_{l} \in V(G): \forall_{v \in V(G)}\left(v_{l}, v\right) \notin E(G)\right\} .
$$

In a similar way the root $R(G)$ can be described as a single node without predecessors

$$
\exists !_{R(G)} R(G) \in V(G) \wedge \forall_{v \in V(G)}(v, R(G)) \notin E(G) .
$$

The graph, which collects all recognized classes can be used as an ensemble of classifiers. Each node, which is not a leaf $v \notin L(G)$, is connected with a classifier. The decision of classifier shows the next node in the graph $c(v)=u$. Finally, the decision determines a leaf. When the process starts at the root then the final leaf determines the recognized class $c(v)=C_{i}$. The decision process can be given as a recursive function

$$
C(v)=\left\{\begin{array}{ll}
C(u), & \text { if } v \notin L(G) \wedge c(v)=u \\
C_{i}, & \text { if } v \in L(G) \wedge c(v)=C_{i}
\end{array} .\right.
$$

The set of classes $C_{i}$, which is equated with the graph $G$, can be described as

$$
C(G)=\left\{C_{i}: c\left(v_{l}\right)=i \wedge v_{l} \in L(G)\right\} .
$$




\subsection{Similarity}

A similarity between classes is estimated on the base of a distance. The distance between classes $d\left(C_{X}, C_{Y}\right)$ depends on the distance between elements of those classes $d(x, y)$ and can be defined as the distance between nearest elements

$$
d\left(C_{X}, C_{Y}\right)=\min _{\substack{x \in C_{X} \\ y \in C_{Y}}} d(x, y),
$$

furthest elements

$$
d\left(C_{X}, C_{Y}\right)=\max _{\substack{x \in C_{X} \\ y \in C_{Y}}} d(x, y)
$$

or as the average distance between all pair of elements in the two different classes

$$
d\left(C_{X}, C_{Y}\right)=\frac{1}{n_{C_{X}} n_{C_{Y}}} \sum_{\substack{x \in C_{X} \\ y \in C_{Y}}} d(x, y) .
$$

All those methods need calculation of the distance between all pair of elements. When the classes have too many members the distance may be approximated as the distance between centroids (the centers of gravity for the classes)

$$
d\left(C_{X}, C_{Y}\right)=d\left(\frac{1}{n_{C_{X}}} \sum_{x \in C_{X}} x, \frac{1}{n_{C_{Y}}} \sum_{y \in C_{Y}} y\right) .
$$

In a similar, way a distance between groups of classes can be calculated. If a group is an union of classes $C_{X}=\bigcup_{i=1}^{k} C_{i}$ then all members of classes $C_{i}$, where $i=1 \ldots k$, are treated as members of $C_{X}$. The distance between such groups can be calculated as (5), (6), (7) or (8). A single class may be compared with a group of classes in the same way.

The distance between an individual elements of the data space $d(x, y)$ depends on the selected metric. Usually it is the Euclidean metric

$$
d(x, y)=\sqrt{\sum_{i=1}^{n}\left(x_{i}-y_{i}\right)^{2}} .
$$

However, if the recognized elements are described by some specific features it is sometime better to select a different measure.

Two potential candidates are Manhattan and Chebyshev metrics. the Manhattan distance

$$
d(x, y)=\sum_{i=1}^{n}\left|x_{i}-y_{i}\right|
$$

should be calculated when individual features are independent, but their sum may be reated as a rational measure of the similarity. In the Chebyshev distance

$$
d(x, y)=\max _{i \in\{1, \ldots, n\}}\left|x_{i}-y_{i}\right|
$$

the similarity will depend on the maximal difference among features. 


\section{$3.3 \quad$ Structure}

The graph for the $n$-classes classification task has to group $n$ leaves. The algorithm for creating such graph starts with the set $S_{G}$ of $n$ graphs with only one node

$$
S_{G}=\left\{G_{i}: C\left(G_{i}\right)=\left\{C_{i}\right\} \wedge i=1 \ldots n\right\} .
$$

In each step a pair $\left(G_{i}, G_{j}\right)$ of graphs equivalent with the nearest sets of classes is found

$$
\left(G_{i}, G_{j}\right)=\arg \min _{G_{i}, G_{j} \in S_{G}} d\left(C\left(G_{i}\right), C\left(G_{j}\right)\right) .
$$

Both, $C\left(G_{i}\right)$ and $C\left(G_{j}\right)$ are sets of classes and the distance $d\left(C\left(G_{i}\right), C\left(G_{j}\right)\right)$ can be calculated as (5), (6), (7) or (8).

The graphs $G_{i}$ and $G_{j}$ are joined into a single graph $G$. In the joining process a new node $v$ is added as the root (2) for the new graph. The roots of joined graphs become successors of the new node and the new graph $G$ is given by the set of nodes

$$
V(G)=\{v\} \cup V\left(G_{i}\right) \cup V\left(G_{j}\right)
$$

and the set of edges

$$
E(G)=\left\{\left(v, R\left(G_{i}\right)\right),\left(v, R\left(G_{j}\right)\right)\right\} \cup E\left(G_{i}\right) \cup E\left(G_{j}\right) .
$$

The newly created graph $G$ is added to the set of graphs

$$
S_{G}=S_{G} \cup\{G\} .
$$

The component graphs $G_{i}$ and $G_{j}$ are not removed from the set $S_{G}$. The graphs are equivalent to classes $C_{i}$ and $C_{j}$ respectively. However, on the base of (4) and (14), (15), the graph $G$ is equated with the set $\left\{C_{i}, C_{j}\right\}$. For that reason the condition (13) has to choose $\left(G_{i}, G\right)$ or $\left(G, G_{j}\right)$ as the nearest graphs. To avoid such situation possibilities of joining have to be limited. Two graphs can be joined if and only if the set of classes represented by one of them (4) is not a subset of the other

$$
C\left(G_{i}\right) \nsubseteq C\left(G_{j}\right) \wedge C\left(G_{j}\right) \nsubseteq C\left(G_{i}\right) .
$$

If the new created graph will not be one of the nearest graphs then graphs $G_{i}$ and $G_{j}$ are still the nearest ones. In such situation the algorithm will not stop. For that an additional condition has to be formed. The two graphs can be joined if and only if the union of classes represented by them is not represented by any existed graph

$$
\forall_{G \in S_{G}} C(G) \neq C\left(G_{i}\right) \cup C\left(G_{j}\right) .
$$


Both conditions (17) and (18) can be used to create a limited set of allowed pairs of graphs

$$
\begin{aligned}
S_{P} & =\left\{\left(G_{i}, G_{j}\right): G_{i}, G_{j} \in S_{G}\right. \\
& \wedge C\left(G_{i}\right) \nsubseteq C\left(G_{j}\right) \wedge C\left(G_{j}\right) \nsubseteq C\left(G_{i}\right) \\
& \left.\wedge \forall_{G \in S_{G}} C(G) \neq C\left(G_{i}\right) \cup C\left(G_{j}\right)\right\} .
\end{aligned}
$$

Moreover, the common part of classes is ignored when the distance is calculated and the final form of the formula (13) is

$$
\left(G_{i}, G_{j}\right)=\arg \min _{G_{i}, G_{j} \in S_{P}} d\left(C\left(G_{i}\right) \backslash C\left(G_{i} \cap G_{j}\right), G_{j} \backslash C\left(G_{i} \cap G_{j}\right)\right),
$$

where

$$
C\left(G_{i} \cap G_{j}\right)=C\left(G_{i}\right) \cap C\left(G_{j}\right) .
$$

In each step of the algorithm, the two allowed graphs $G_{i}, G_{j}$ are joined. The algorithm stops when no join can be made

$$
\forall_{G_{i} \in S_{G}} \exists_{G_{j} \in S_{G}} C\left(G_{i}\right) \subseteq C\left(G_{j}\right) \vee C\left(G_{j}\right) \subseteq C\left(G_{i}\right) .
$$

In such case, the set contains one graph $G$, which collects all recognized classes

$$
C(G)=\left\{C_{i}: i=1 \ldots n\right\} \text {. }
$$

In the final graph $G$ some of nodes may be repeated. However, such nodes are leaves or have the same branches and they can be treated as the one. An example of the structure creation process is presented in Fig 1.

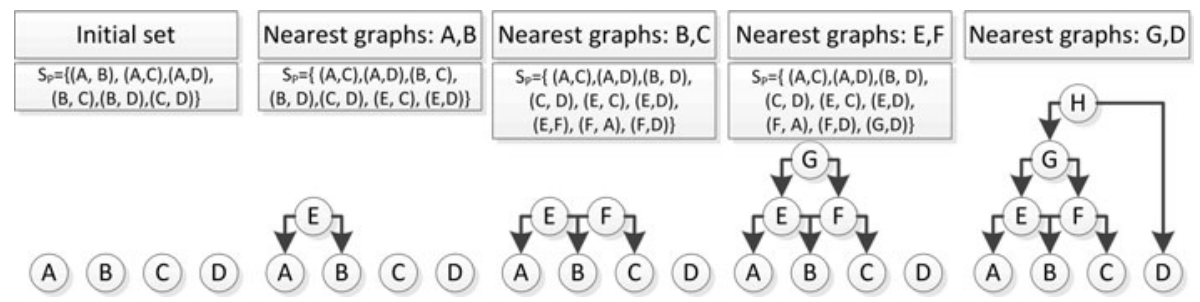

Fig. 1. An example of the creation of the graph structure

\subsection{Classifiers}

In the DAGSVM each classifier rejects one from the recognized classes. In created structure the number of classifiers may be reduced. For that reason the classifier may reject more than one class. 
When a classifier is connected with the node $v$ the function $c(v)$ has to choose one of the successors $u, w$. Each of them is a root of a graph. When the node $u$ is the root of the graph $G_{u}$ then the classification function (3) can only reach classes from the set $C\left(G_{u}\right)$. The same with the node $w$, which represents classes from the set $C\left(G_{w}\right)$. So, the classifier will reject classes

$$
\operatorname{Reject}(v)=\left\{\begin{array}{ll}
C\left(G_{u}\right), & \text { if } c(v)=w \\
C\left(G_{w}\right), & \text { if } c(v)=u
\end{array} .\right.
$$

The classifier connected with the node $v$ will be trained to separate members of the classes includes in the set $C\left(G_{u}\right)$ from those belonging to the set $C\left(G_{w}\right)$.

However, in the directed acyclic graph, two different nodes can have the same successors. In such case, the function (24) has to be modified to avoid discrimination between members of the same class. The new formula of the rejection function will be

$$
\operatorname{Reject}(v)=\left\{\begin{array}{ll}
C\left(G_{u}\right) \backslash C\left(G_{u} \cap G_{w}\right), & \text { if } c(v)=w \\
C\left(G_{w}\right) \backslash C\left(G_{u} \cap G_{w}\right), & \text { if } c(v)=u
\end{array} .\right.
$$

Now, the classifier connected with the node $v$ will be trained to separate members of classes from the set $C\left(G_{u}\right) \backslash C\left(G_{u} \cap G_{w}\right)$ with the members of classes from the set $C\left(G_{w}\right) \backslash C\left(G_{u} \cap G_{w}\right)$ and the problem with discrimination of the same classes will be prevented.

\subsection{Discussion}

The reduced graph may be compared with the DAGSVM in three aspects. The first one is the recognition accuracy. An increase of the accuracy, when the number of the classifiers is reduced, should not be expected. However, it can stay on the similar level, because of limitation of reduction to the classifiers for the distant classes.

Main expectations lie in the reduction of costs. There are two types of the costs: the learning time and the classification time. The classification time is a function of the average number of classifiers. For the DAGSVM this number is constant and equals $n-1$ for $n$-recognized classes. If at least one classifier is removed then the classification cost will be reduced for at least one class. Then the average cost will also be reduced.

The aspect of the learning time is a little more complicated. The number of classifiers, which have to be created is reduced. However, the classification task for a single classifier becomes complex, when the split between groups is examined. The optimization function [11, which calculates coefficients of the discriminant plane, depends on the number of support vectors. Then the number of used SV may be used as an estimator of the learning cost.

The learning process will be faster if the number of the support vectors, which have been used by the rejected classifiers is greater than the number of additional vectors for the new discrimination task. It has to be also noticed that the number 
of the support vectors does not influence the classification time, which will be constant for each SVM classifier.

\section{Classification of Digits Using Grouping Trees}

The digits recognition task was chosen for testing. Handwritten digits were described with 197 features. The feature set is based on a set of projections, histograms and other fast computed features [9. The feature vectors create ten classes. The sizes of classes are unbalanced. Nearly 30 percent of the whole data set is represented by the class 3. This follows from a specific character of the collected data. The digits were extracted from control points on the geodetic maps [10] dominated by thirties. From 7081 collected digits, 5291 were used as a learning probe. The rest, 1790, were used for the tests.

The results for the reduced graph was compared to the results of the OneAgainst-One strategy. A similar classification error (a percentage of mistaken cases) was expected. Also, the learning time as well as classification time were compared. Those two aspect were estimated by a number of support vectors and the number of binary classifiers respectively.

An additional test was for the One-Against-All strategy. This strategy uses a minimal number of classifiers and it should give a reference to the reduction effects. The method was implemented by a tree. The tree, which was created by adding the nearest class to the existing tree. The tree is initiated by two nearest classes.

In all tests SVMs with linear kernels, were chosen. The distances between elements were calculated in the Euclidean metric (9). The tree was built on the base of the average distance (7) and in case of the reduced DAG, the distance between centroids (8) was used.

Table 1. Comparison of methods

\begin{tabular}{|l|c|c|c|c|c|}
\hline Classifier & $\begin{array}{c}\text { Number } \\
\text { of SVM }\end{array}$ & $\begin{array}{c}\text { Average } \\
\text { No. of SVM }\end{array}$ & $\begin{array}{c}\text { Number } \\
\text { of SV }\end{array}$ & $\begin{array}{c}\text { Average } \\
\text { No. of SV }\end{array}$ & $\begin{array}{c}\text { Classification } \\
\text { error }\end{array}$ \\
\hline DAGSVM & 45 & 9 & 2176 & 48 & 4.25 \\
\hline Reduced graph & 26 & 7 & 1600 & 62 & 4.75 \\
\hline OAA & 9 & 5 & 1414 & 157 & 6.65 \\
\hline
\end{tabular}

The results are presented in the Table 1. For each method, the number of used classifier is given as well as the average number of classifiers used in the single classification process. This value allows to estimate the classification time. For the estimation of the learning time a number of support vector is given. The average number of SV is calculated for the single classifier. Finally, the classification error is presented. 
The One-Against-One strategy needs 45 classifiers. When the classifiers are grouped in the DAGSVM the average number of used classifiers is 9 for each data distribution. The classification rate, calculated as a percent of positive recognized elements is 95.75 percent.

The One-Against-All strategy requires only 9 classifiers. The average number of used classifiers is about 5 for the balanced case (the number of elements among classes is similar). The method produces a lower classification rate, 93.35 percent of positive recognized elements. However, the method is relatively fast even in the learning process. The effect of increasing of the average number of support vectors is mitigated by the reduce in the number of created classifiers.

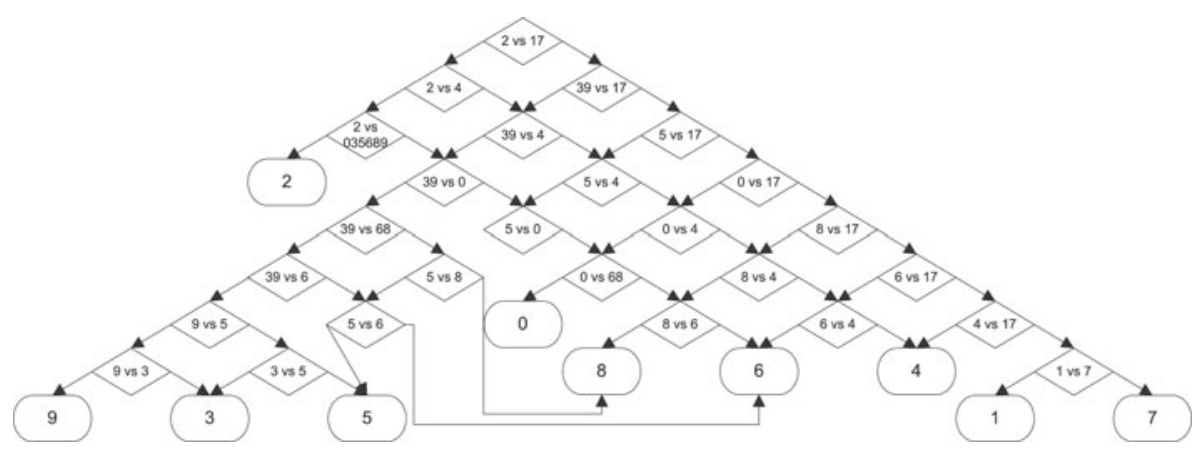

Fig. 2. The reduced DAGSVM for the digits recognition task

The reduced graph (Fig 2) needs 26 classifiers. The average number of used classifiers is 7 in the balanced case. However, some classes, such as 2 , could be reached with 3 classification decisions. The maximal number of used classifiers is 8 . The classification process will be definitely faster than for the DAGSVM. At the same time the classification rate is 95.25 percent of positive recognized cases, which is nearly the same as for the One-Against-One strategy.

The last aspect is the learning time. In the reduced graph the global number of support vectors is lower than in the DAGSVM. The average number of SV grows, but many classifiers are eliminated. The increase of SV is not significant, because most of the classifiers split only two classes as in the case of the DSGSVM.

As a conclusion of the test it can be said that the fastest method is OneAgainst-All (in both the learning time and the classification time). However, the classification error for this method grows in comparison to the One-AgainstOne strategy. Yet, the One-Against-One strategy is not the best method. The reduced graph has a similar classification rate, but the learning and classification costs are significantly lower. 


\section{Summary}

In this paper a new method, which creates the ensemble of the SVM was presented. The method is based on the DAGSVM. However, it allows to reduce a number of created classifiers. The classifiers, which discriminate distant classes are replaced by the classifiers, which separate groups of classes. The algorithm for creating a new graph structure, was presented.

The algorithm was tested on the handwritten digits recognition task. The recognition rate stays similar to the DAGSVM method, while the reduction of the classifiers was significant. The reduction of the average number of classifiers decreases the classification time. Moreover, the learning time should not be increased despite of increase of the average number of support vectors used by the classifiers.

\section{References}

1. Abe, S.: Support Vector Machines for Pattern Classification (Advances in Pattern Recognition). Springer-Verlag New York, Inc., Secaucus (2005)

2. Kumar, M.A., Gopal, M.: A comparison study on multiple binary-class SVM methods for unilabel text categorization. Pattern Recogn. Lett. 32, 9160:311-9160:323 (2010), http://dx.doi.org/10.1007/s11063-010-9160-y

3. Bennett, K.P.: Combining support vector and mathematical programming methods for classification, pp. 307-326. MIT Press, Cambridge (1999)

4. Crammer, K., Singer, Y.: On the learnability and design of output codes for multiclass problems. In: COLT 2000: Proceedings of the Thirteenth Annual Conference on Computational Learning Theory, pp. 35-46. Morgan Kaufmann Publishers Inc., San Francisco (2000)

5. Kijsirikul, B., Ussivakul, N., Meknavin, S.: Adaptive directed acyclic graphs for multiclass classification. In: Ishizuka, M., Sattar, A. (eds.) PRICAI 2002. LNCS (LNAI), vol. 2417, pp. 158-168. Springer, Heidelberg (2002)

6. Kim, H.-C., Pang, S., Je, H.-M., Kim, D., Bang, S.Y.: Constructing support vector machine ensemble. Pattern Recognition 36(12), 2757-2767 (2003)

7. Kressel, U.H.G.: Pairwise classification and support vector machines, pp. 255-268. MIT Press, Cambridge (1999)

8. Platt, J., Cristianini, N., ShaweTaylor, J.: Large margin dags for multiclass classification. In: Solla, S.A., Leen, T.K., Mueller, K.-R. (eds.) Advances in Neural Information Processing Systems 12, pp. 547-553 (2000)

9. Romero, R., Touretzky, D., Thibadeau, R.: Optical chinese character recognition using probabilistic neural networks. Pattern Recognition 8, 1279-1292 (1997)

10. Stapor, K.: Geographic map image interpretation - survey and problems. Machine Graphics \& Vision 9(1/2), 497-518 (2000)

11. Vapnik, V.: The Nature of Statistical Learning Theory. Springer, Heidelberg (1995)

12. Wilson, R.J.: Introduction to graph theory. John Wiley \& Sons, Inc., New York (1986)

13. Ye, W., Shang-Teng, H.: Reducing the number of sub-classifiers for pairwise multicategory support vector machines. Pattern Recogn. Lett. 28, 2088-2093 (2007) 